\title{
Philosophiques
}

\section{Silvia Benso, Viva Voce. Conversations with Italian Philosophers, New York, SUNY Press, 2017, 308 pages}

\section{Firmin Havugimana}

Volume 45, numéro 1, printemps 2018

URI : https://id.erudit.org/iderudit/1048636ar

DOI : https://doi.org/10.7202/1048636ar

Aller au sommaire du numéro

Éditeur(s)

Société de philosophie du Québec

ISSN

0316-2923 (imprimé)

1492-1391 (numérique)

Découvrir la revue

Citer ce compte rendu

Havugimana, F. (2018). Compte rendu de [Silvia Benso, Viva Voce.

Conversations with Italian Philosophers, New York, SUNY Press, 2017, 308

pages]. Philosophiques, 45(1), 334-338. https://doi.org/10.7202/1048636ar d'utilisation que vous pouvez consulter en ligne.

https://apropos.erudit.org/fr/usagers/politique-dutilisation/ 


\section{Silvia Benso, Viva Voce. Conversations with Italian Philosophers, New York, SUNY Press, 2017, 308 pages.}

Dix ans après Contemporary Italian Philosophy. Crossing the Borders of Ethics, Politics, and Religion ${ }^{1}$, Silvia Benso convie à nouveau dix-sept philosophes contemporains, italiens et italiennes, à partager leurs contributions théoriques avec le lectorat anglophone. La collection d'entretiens que constitue Viva Voce signe ainsi une décennie de vie éditoriale pour la collection des SUNY Press consacrée à la philosophie italienne actuelle, et note dès son introduction la reconnaissance croissante que cette dernière a pu gagner dernièrement ${ }^{2}$. Le panorama offert par Benso maintient la focale sur la philosophie dite continentale, mais l'autrice consacre cette fois-ci une section à des acteurs dont les recherches relèvent plutôt de la tradition analytique (Evandro Agazzi, Giulio Giorello et Paolo Parrini dans «Rationalité, Sciences, Expérience $\left.{ }^{3} »\right)$. Après tout, dans sa préface de 2007, Silvia Benso reconnaissait déjà l'incontestable diversité de la philosophie transalpine: pour les $\mathrm{xx}^{\mathrm{e}}$ et $\mathrm{XXI}^{\mathrm{e}}$ siècles, on avait répertorié une cinquantaine de philosophes, dans des champs aussi variés que le néopositivisme, le néoidéalisme, l'herméneutique, le marxisme, l'existentialisme, l'histoire de la philosophie, la phénoménologie, l'esthétique ou encore la philosophie du langage et des sciences.

Dans le cadre de ce compte rendu, nous proposons toutefois de nous limiter aux entretiens de la philosophe Adriana Cavarero ${ }^{4}$ et de l'herméneute Gianni Vattimo ${ }^{5}$, ainsi que sur certaines observations critiques de Benso quant au nouvel intérêt porté à la philosophie italienne. Bien que Cavarero et Vattimo soient classés au sein de la même section thématique ( «Histoire, Justice, Communautés »), ce choix de notre part est simplement un compromis fait aux écueils évidents qui attendent une introduction globale à la philosophie italienne qui se voudrait en même temps exhaustive. Il nous apparaît alors plus pertinent de céder la parole à la seule contributrice

1. Silvia Benso et Brian Schroeder, dir., Contemporary Italian Philosophy, New York, SUNY Press, 2007, 306 pages.

2. La même chose pourrait être observée au sein de la francophonie, notamment avec la publication des actes du colloque international de 20I4 qui s'est tenu à la Sorbonne et à l'Université Paris Ouest Nanterre La Défense: Silvia Contarini et Davide Luglio, dir., L'Italian Theory existe-t-elle?, Paris, Mimesis, 2015, 2 Io pages.

3. Nous traduisons les intitulés des sections thématiques de l'ouvrage et traduirons les citations directes des textes anglais et italien.

4. Cavarero est née dans le Piémont en I947 et réside aujourd'hui à Vérone. Son travail touche à la philosophie politique, dont ses œuvres en théorie féministe sur la différence sexuelle, et à la littérature.

5. Également né dans le Piémont, mais en I936, Vattimo est un philosophe que l'on tend à ranger du côté des postmodernistes. Il s'est aussi engagé dans une carrière politique, cumulant deux mandats au Parlement européen. 
du recueil d'entretiens ${ }^{6}$ et au plus traduit, en français du moins, des philosophes participant à ces interviews. Notons tout de même que les cinq autres sections thématiques touchent autant à la philosophie de la religion ( «Êtres humains, Mal, Transcendance»), à la métaphysique ( «Être, Néant, Temporalité, Lieu»), qu'à l'esthétique («Imagination, Art, Technologie»), ou à l'éthique ( «Éthique, Passions, Pratiques»).

Les interviews menées par Benso invitent les philosophes convoqués à dresser un portrait d'eux- et elles-mêmes, et suivent la même structure. Leur parcours universitaire et militant, les textes et les thèmes philosophiques qui ont pu les marquer, ainsi que la plus représentative de leurs œuvres selon eux, sont tout autant abordés dans les entretiens.

Dans le cas d'Adriana Cavarero, la discussion l'amène à rappeler comment l'esprit soixante-huitard était présent en I97 I lorsqu'elle obtint son diplôme de l'Université de Padoue. Après qu'elle eut reçu une éducation classique qui l'avait initiée aux textes de Platon, Hobbes et Hegel, c'est la propagation d'une culture antiautoritaire et en rupture avec la tradition au sein des universités italiennes qui semble l'avoir le plus stimulée. Cette "contre-culture» passait par la découverte de Foucault, Beauvoir, et l'École de Francfort, mais aussi par la relecture de Gramsci et de Luckács. Le «vent révolutionnaire $^{7}$ " qui soufflait au cours des années soixante-dix accompagna ainsi son implication au sein d'associations féministes, et c'est dans les années quatre-vingt qu'elle créera à Vérone, avec Luisa Muraro ${ }^{8}$, la Comunità filosofica di Diotima. Luce Irigaray est alors une grande inspiration pour les membres de Diotima, et la question de la différence sexuelle est au cœur de leurs préoccupations. Aux yeux de Cavarero, c'est son Nonostante Platone 9 qui témoigne du travail intense fait au sein de la communauté philosophique véronaise. Aux côtés de Platon, l'autrice reconnait également Hannah Arendt comme inspiration majeure, ne serait-ce que pour sa subversive attention philologique aux textes et sa tentative d'élaborer une ontologie relationnelle capable de passer outre les impasses du solipsisme. Enfin,

6. Notons que la nette prépondérance d'hommes interviewés est soulevée et déplorée par Silvia Benso dans son introduction. Cette dernière explique cet état de fait par la constitution de la scène philosophique italienne même: les femmes y peinent encore à accéder à des postes au sein des universités et à voir la spécificité, la pertinence et la force de leur travail reconnue. La sélection de Benso reposant sur une reconnaissance académique des philosophes choisis contrevient ainsi à un échantillon au moins paritaire de philosophes italiens et italiennes. Voir Silvia Benso, Viva Voce, New York, SUNY Press, 20I7, p. Iо.

7. Ibid, p. 73 .

8. Notons que la collection éditée par Benso publiera en 2018 une traduction anglaise de L'ordine simbolico della madre. Le lecteur francophone pourra en consulter la traduction française: Luisa Muraro, L'ordre symbolique de la mère, Paris, L'Harmattan, 2003, I62 pages.

9. On pourrait traduire le titre entier par Malgré Platon. Figures féminines dans la philosophie antique. L'ouvrage n'est pas traduit en français, mais l'est en anglais: Andrea Cavarero, In Spite of Plato, London, Routledge, I995, I35 pages. 
c'est Tu che mi guardi, tu che mi racconti ${ }^{10}$, un texte des années quatrevingt-dix écrit après son départ de Diotima, que Cavarero propose comme étant le plus représentatif de ses œuvres. Cet ouvrage s'attarde aux tensions envisageables entre l'universalité abstraite de la philosophie, comprise comme discipline et discours sur le quoi, et le particularisme singulier des narrations biographiques, concernées par le qui du protagoniste raconté. Ce texte poursuit ainsi un geste que la philosophe appréciait chez Arendt: une façon de mélanger pensée politique, philosophie et littérature.

L'entretien avec Gianni Vattimo suit le même cours. En guise de conclusion à son entretien, le philosophe hésite entre deux de ses textes quand il est convoqué à proclamer l'opus magnum de sa carrière. Le premier est Il sogetto e la maschera, un commentaire de Nietzsche des années soixante-dix auquel Vattimo pense toujours, tandis que le second est son dernier livre: Della realtà ${ }^{11}$. Aux yeux de l'herméneute, c'est la façon dont il a pu libérer la différence ontologique heideggérienne de son poids métaphysique en l'historicisant qui est centrale à son travail. Le philosophe considère alors son " "heiddeggérianisme de gauche" 12 » comme crucial afin de penser la technicisation généralisée du monde à l'ère du capitalisme tardif. C'est donc à une herméneutique communiste qu'il convie en rapprochant son travail des préoccupations qui étaient celles d'Adorno et de l'École de Francfort. S'expliquant sur son intérêt pour Nietzsche et Heidegger, il signale aussi que tout son travail a été attaché à la question du nihilisme et que même sa notion de pensiero debole ${ }^{13}$, plutôt tributaire du travail de René Girard sur la religion, est redevable à sa minutieuse étude des deux auteurs allemands. Ailleurs, le philosophe rappelait que cette théorie de la "pensée faible" tâchait de thématiser en quoi le sens de l'être se traduisait par un affaiblissement de la philosophie ${ }^{14}$. Enfin, Gianni Vattimo rappelle qu'il a été élève de Luigi Pareyson et que sa formation philosophique doit beaucoup à l'existentialisme catholique du philosophe piémontais. C'est dans le contexte de reconstruction qui a fait suite à la Deuxième Guerre mondiale que Vattimo poursuit ses études, et c'est en tant que militant catholique et communiste (cattocommunista) qu'il évolue au sein d'une université italienne qui délaisse peu à peu les débats «italocentrés» de Benedetto Croce et Giovanni Gentile pour s'intéresser à Dewey, Wittgenstein ou encore au Cercle de Vienne. Sans oublier le fait que le marxisme a une présence notable

10. La traduction anglaise est disponible sous le titre suivant: Adriana Cavarero, Relating Narratives, London, Routledge, 2000, I 84 pages.

11. Des deux, seul Della realtà est traduit, et en anglais: Gianni Vattimo, On Reality, New York, Columbia University Press, 20I7, 248 pages.

12. Viva Voce, New York, p. I Iо.

13. Voir Gianni Vattimo, Weak Thought, New York, SUNY Press, 20I 2, 277 pages.

14. Voir Anne Staquet, La pensée faible de Vattimo et Rovatti: une pensée-fable, Paris, L'Harmattan, I996, p. I66. 
dans les années cinquante, mais que c'est plutôt une division entre laïques (laicisti) et catholiques qui règne alors au sein de l'université.

Signalons en terminant que les mots d'ouverture de Viva Voce sur la reconnaissance grandissante de la philosophie italienne sont une réponse encourageante à ceux avec lesquels la préface de Contemporary Italian Philosophy débutait: «La prédominance de la pensée française et allemande en philosophie continentale contemporaine en est malheureusement venue à éclipser, sinon véritablement exclure, beaucoup de pensées de l'Europe méditerranéenne ${ }^{15}$.»

Dans Viva Voce, Benso remarque cette fois-ci qu'au cours des dix dernières années, avec Vattimo et Cavarero, des philosophes comme Giorgio Agamben, et Toni Negri - mais aussi d'autres comme Noberto Bobbio, Mario Perniola et Carlo Sini - ont bénéficié de traductions anglaises qui les ont aidés à gagner en popularité. Toutefois, Benso ajoute que la chose est plutôt due à des événements et des circonstances contingentes qu'à un effort concerté pour diffuser et apprécier la philosophie italienne du fait de la richesse de ses textes et de son contexte culturel. Cette remarque critique ne l'amène aucunement à verser dans un nationalisme douteux, mais plutôt à penser à l'écart de la thèse du philosophe Roberto Esposito ${ }^{16}$. S'inspirant du concept deleuzo-guattarien de déterritorialisation, ce dernier propose de penser le propre de la philosophie italienne sur le mode d'une fuite déterritorialisante, de telle sorte que son développement impliquerait non seulement une décontextualisation territoriale (l'émigration de certains philosophes italiens vers l'Amérique ou la France), mais aussi une décontextualisation disciplinaire (via un intérêt pour le non philosophique). Autrement dit, si un certain rapport du dedans au dehors pourrait décrire l'essence de la philosophie italienne, ce serait une continuelle fuite, comprise comme une lancée vers son dehors. Pour cette raison, Silvia Benso propose plutôt de penser le rapport de la philosophie italienne à son dehors sur le mode d'une porosité hospitalière. Si la philosophie italienne tend à un mouvement vers une extériorité à elle-même, c'est en tant que cette extériorité est reçue et acceptée en son sein dans un geste d'ouverture toujours constructif. Ce rapport au dehors ne serait donc pas à lire comme une fuite qui traduirait une incapacité à l'autonomie.

En somme, le recueil d'entretiens de Silvia Benso, et le travail général de publicisation mené par elle et d'autres auprès des SUNY Press, avec la collection SUNY Series in Contemporary Italian Philosophy, sont remarquables. On déplorera que la majorité des philosophes interviewés et traduits demeurent des hommes, d'autant plus que le précédent recueil d'entretiens de Silvia Benso pointait vers les œuvres de philosophes contem-

15. Silvia Benso et Brian Schroeder, dir, Contemporary Italian Philosophy, p. ix.

16. Voir Roberto Esposito, Living Thought. The Origins and Actuality of Italian Philosophy, Redwood City, Stanford University Press, 20I 2, 296 pages. 
poraines, comme Silvana Borutti, Donatella di Cesare, ou Chiara Zamboni, pour ne nommer que celles-là. En dépit de cela, Viva Voce représente un outil de recherche des plus bienvenus, qui permet à la fois de situer historiquement la pensée des philosophes italiennes et italiens, et de mettre en lumière la genèse historique de certains de leurs concepts. Sans oublier que cet ouvrage recèle un index et une excellente bibliographie sélective des philosophes interviewés. Il constitue en ce sens une admirable propédeutique à la philosophie italienne et une piste à suivre pour les chercheurs et chercheuses.

FIRMIN HAVUGIMANA

Université Paris-8 Vincennes-Saint-Denis 